linien zu behandeln. Dazu gehört der richtige Einsatz von LCZ696 - für Patienten mit und ohne Diabetes - wenigstens bis wir mehr Daten dazu haben.

Das macht Medizin spannend. Nicht das Dogma des weißhaarigen Professors, sondern die Bewertung des zeitgemäßen, wandelbaren medizinischen Wissens sollte unsere Entscheidungen führen. Und trotz oder gerade wegen aller Fortschritte brauchen wir gesunden Menschenverstand oder „common sense“ aber nicht nur, dies wäre ein Schritt zurück. Die Vielfalt der (medizinischen) Daten, Guidelines, Kommentare und Bewertungen verführt dazu, den Patienten als Mensch zu übersehen. Wenn wir das Bewährte anwenden (ACE-Hemmer, Betablocker und Mineralokortikoidantagonisten in maximal tolerierter Dosis) werden wir den Nutzen einer zusätzlichen Therapie mit LCZ696 erkennen können - nach aktueller Datenlage für Nichtdiabetiker und auch für Diabetiker! Dies sollte common sense sein. - Und: Common sense ist für alle wichtig - er ist die Grundlage der Demokratie.
Literatur:

1. http://eurheartj.oxfordjournals.org/content/37/27/2129

2. Gilbert RE, Krum H. Lancet. 2015 May 23;385(9982):2107-17.

3. McMurray JJ, Packer M, Desai AS et al. N Engl J Med 2014:371(11):993-1004.

4. Kristensen SL et al. Circ Heart Fail. 2016 Jan;9(1). pii: e002560.

5. Schulz KF, Grimes DA. Lancet. 2005 May 7-13;365(9471):1657-61.

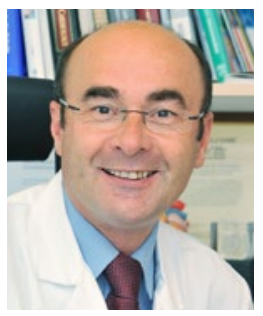

Prof. Dr. med. Robert HG Schwinger

Chefarzt - Med. Klinik II, Kliniken Nordoberpfalz AG, Klinikum Weiden, Akademisches Lehrkrankenhaus der Universität Regensburg Söllnerstr.16, 92637 Weiden i.d. OPf.

Robert.Schwinger@Kliniken-Nordoberpfalz.ag

\title{
Jetzt mit pfiffigen Ideen bewerben
}

Wie lassen sich Patienten besser versorgen, wie lässt sich die Arztpraxis wirtschaftlich optimieren? Mediziner, die hierauf eine praxistaugliche Antwort gefunden haben, können sich jetzt bewerben und auf den Praxis-Preis 2016 hoffen.

"Lassen Sie Ihre Ideen fliegen“ - unter diesem neuen Motto suchen die Fachverlagsgruppe Springer Medizin, zu der auch „Info Diabetologie“ und die „Ärzte Zeitung“ gehören, und UCB Innere Medizin jetzt Erfolgsrezepte für die Arztpraxis 2016. Etwa solche, die dabei helfen, die Arbeit als Arzt zu vereinfachen, um so Patienten besser versorgen zu können. Abheben können beim Wettbewerb gute Ideen aus allen Bereichen der Arbeit in einer Arztpraxis - es muss dabei auch nicht immer gleich die Welt neu erfunden werden, auch kleine Ideen in der Praxis haben eine Chance. Bürokratie, Ärztemangel, Qualitätsmanagement, Delegation, Compliance, Marketing, Abrechnung, Mitarbeiterführung und der Kampf gegen Volkskrankheiten wie Diabetes und Bluthochdruck - die Themenbandbreite in denen Mediziner innovative Ansätze entwickeln können, um das Unternehmen Arztpraxis zu optimieren, erscheint unerschöpflich. Haben Sie z.B. ein konkretes Konzept entwickelt, das die Wartezeiten für Patienten verkürzt? Setzen Sie spezielle Techniken ein, um das Praxispersonal motiviert und gesund zu halten? Haben sie eine besondere Kooperation mit Kollegen entwickelt, um den Versorgungsdruck zu vermindern? Haben Sie ein besonderes Konzept ausgetüftelt, um die Adhärenz Ihrer Diabetiker zu stärken? Dann bewerben Sie sich bei uns!

\section{Stichtag ist der 30. November}

Ärzte und auch ihre Praxisteams können bis zum 30. November mitmachen und ihre Idee in den Wettbewerb einbringen.
Wer die unabhängige Jury und die InternetNutzer auf aerztezeitung.de mit seinem Projekt, Konzept oder Geistesblitz zur Optimierung der Versorgung beeindruckt, kann wertvolle Preise gewinnen. Die drei Gewinnerteams stellen ihre Konzepte persönlich bei Springer Medizin in Berlin vor.

1. Preis: Ein dreitägiger Ausbildungslehrgang mit Abschluss ,geprüfte Assistenz für Versorgung und Prävention" (Better Care Assistenz). Zudem haben Teilnehmer die Chance auf Buchpreise von Springer Medizin und HCC.

Bewerbungen mit der Beschreibung der eigenen Idee können unkompliziert per Mail an erfolgsrezept@springer.com gesendet werden. „Die Teilnahme an diesem Wettbewerb legen wir anderen Kolleginnen und Kollegen sehr ans Herz. Wir haben uns dafür entschieden, weil wir denken, dass unser Projekt auch für andere Arztpraxen lebbar und sinnvoll ist", zeigen sich die Mediziner Carla Martin \& Michael Pohling aus Flechtorf in Niedersachesn überzeugt. Sie haben 2015 am Wettbewerb teilgenommen und zählten zu den Gewinnern. Durchsetzen konnten sie sich mit ihrem Konzept einer wohnortnahen, hospizähnlichen Palliativversorgung. Geschaffen haben die Ärzte ein ,gemeinde-internes Palliativnetz", das Menschen das Sterben in Wohnortnähe und in der Umgebung von vertrauten Menschen erlaubt.

Marco Hübner

\section{Anmeldung zum Praxis-Preis 2016 \\ Per Internet: aerztezeitung.de/erfolgsrezept \\ PerE-Mail: erfolgsrezept@springer.com \\ Per Post: Ärzte Zeitung Stichwort: Erfolgs-Rezept - Der \\ Praxis-Preis 2016 Postfach 2131, 63243 Neu-Isenburg}

